\title{
The beta family at the prime two and modular forms of level three
}

\author{
HANNO VON BODECKER
}

\begin{abstract}
We use the orientation underlying the Hirzebruch genus of level three to map the beta family at the prime $p=2$ into the ring of divided congruences. This procedure, which may be thought of as the elliptic Greek letter beta construction, yields the $f$-invariants of this family.
\end{abstract}

55Q45; 11F11, 55Q51, 58J26

\section{Introduction and statement of the results}

The Adams-Novikov spectral sequence (ANSS) serves as a powerful tool to understand the structure of the stable homotopy groups of the sphere $\pi_{*} S^{0}$ : working locally at a fixed prime $p$, we have

$$
\mathrm{E}_{2}^{s, t}=\mathrm{Ext}_{\mathrm{BP}_{*} \mathrm{BP}}^{s, t}\left(\mathrm{BP}_{*}, \mathrm{BP}_{*}\right) \Rightarrow\left(\pi_{t-s} S^{0}\right)_{(p)},
$$

and much insight can be gained by resolving its $E_{2}$-term into $v_{n}$-periodic components (see eg Ravenel's book [11]). In their seminal paper propagating this chromatic approach, Miller, Ravenel, and Wilson [10] introduced the so-called Greek letter map, and computed the 1-line (for all primes) and the 2-line (for odd primes), generated by the alpha and beta families, respectively. The computation of the 2-line for $p=2$, which we outline here, is due to Shimomura [12]. Let us concentrate on the beta elements at $p=2$ (where there are also products of alpha elements). Starting from certain elements $x_{i} \in v_{2}^{-1} \mathrm{BP}_{*}$ and $y_{i} \in v_{1}^{-1} \mathrm{BP}_{*}$, put

$$
a_{0}=1, \quad a_{1}=2, \quad a_{k}=3 \cdot 2^{k-1} \text { for } k \geq 2 ;
$$

then, for $n \geq 0$, odd $s \geq 1, j \geq 1, i \geq 0$, subject to the conditions

$$
n \geq i, \quad 2^{i} \mid j, \quad j \leq a_{n-i}, \quad \text { and } \quad j \leq 2^{n} \text { if } s=1 \text { and } i=0,
$$

the simplest beta elements are given by $[12,(1.3 .1)]$

$$
\beta_{s \cdot 2^{n} / j, i+1}=\eta\left(x_{n}^{s} / 2^{i+1} v_{1}^{j}\right),
$$

where $\eta$ is the universal Greek letter map (see [10, (3.6)]). In fact, it is sometimes possible to improve divisibility: namely, for $n, s, j$, and $i$ as above with the additional 
conditions that

$$
n \geq i+1 \geq 2, \quad j=2 \quad \text { and } \quad s \geq 3 \quad \text { if } n=2, \quad j \leq a_{n-i-1} \quad \text { if } n \geq 3,
$$

Shimomura defines [12, (1.3.2)]

$$
\beta_{s \cdot 2^{n} / j, i+2}=\eta\left(x_{n}^{s} / 2^{i+2} y_{i}^{m}\right), \quad \text { where } m=j / 2^{i},
$$

and shows the following relations between the beta elements given by (1) and (2) [12, Lemma 3.10]:

(i) $\beta_{s \cdot 2^{n} / j, i+2}=\beta_{s \cdot 2^{n} / j,(i+1)+1}$ if $2^{i+1} \mid j$,

(ii) $2 \beta_{s \cdot 2^{n} / j, i+2}=\beta_{s \cdot 2^{n} / j, i+1}$.

There are striking number-theoretical patterns lurking in the stable stems which become visible from the chromatic point of view, eg the (nowadays) well-known relation between the 1-line and the (denominators of the) Bernoulli numbers. Concerning the 2-line, Behrens [1] established a precise relation between the beta family for primes $p \geq 5$ and the existence of modular forms satisfying appropriate congruences. On the other hand, using an injection of the 2-line into the ring of divided congruences (tensored with $\mathbb{Q} / \mathbb{Z}$ ), Laures [9] introduced the $f$-invariant as a higher analog of the $e$-invariant. Subsequent work (see Behrens and Laures [2]) has shown how these approaches can be merged and used to derive the $f$-invariant of the beta family, albeit still only for $p \geq 5$. A different route has been taken by Hornbostel and Naumann in [8], where the $f$-invariant is represented using Artin-Schreier theory; however, although no longer limited to primes $p \geq 5$, the calculations actually carried out in that reference only take care of two subfamilies (namely $\beta_{t}$ for $p \nmid t$ and $\beta_{s 2^{n} / 2^{n}}$ for $p=2$ ).

Since there has been some progress on our geometrical understanding of the $f$-invariant through analytical techniques (see eg work of the author [3] and Bunke and Naumann [5]), it is desirable to have some sort of "comparison table"; to this end, we compute the $f$-invariant of the beta family ${ }^{1}$ at the prime $p=2$. More precisely, we take a look at the following diagram for $p=2$ and $N=3$ :

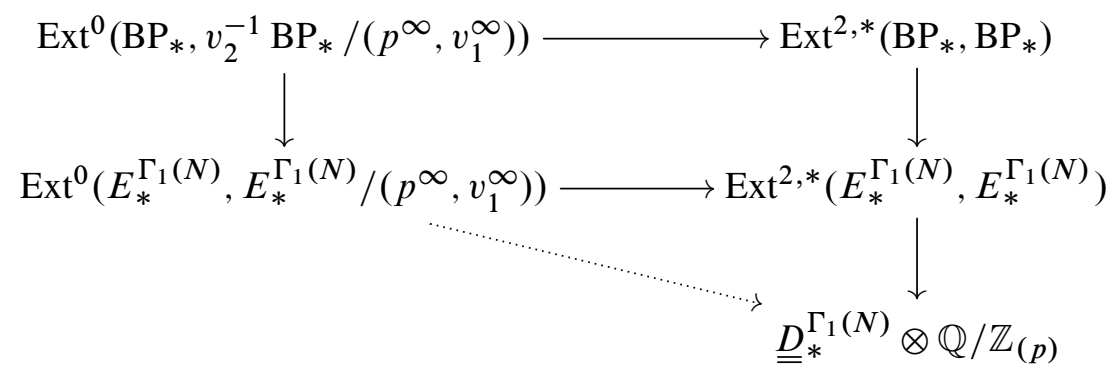

${ }^{1}$ The situation of products of permanent alpha elements has been studied in [4]. 
The upper horizontal arrow in this diagram produces the beta family; as a brief reminder of this construction (referring to $[10 ; 12 ; 11]$ for details), note that an element of this family is annihilated by some power of 2 , say $2^{i_{0}}$. Thus, it is the image of an element in $\operatorname{Ext}^{1}\left(\mathrm{BP}_{*}, \mathrm{BP}_{*} /\left(2^{i_{0}}\right)\right)$ under the connecting homomorphism associated to the short exact sequence $\mathrm{BP}_{*} \rightarrow \mathrm{BP}_{*} \rightarrow \mathrm{BP}_{*} /\left(2^{i_{0}}\right)$. As this Ext ${ }^{1}$-group admits multiplication by (suitable powers of) $v_{1}$, the same argument shows that its $v_{1}^{i_{1}}$-torsion elements can be obtained from $\operatorname{Ext}^{0}\left(\mathrm{BP}_{*}, \mathrm{BP}_{*} /\left(2^{i_{0}}, v_{1}^{i_{1}}\right)\right)$. In particular, it turns out that, under the conditions quoted before, $x_{n}^{s}$ is invariant $\bmod \left(2^{i+1}, v_{1}^{j}\right)$, thus giving rise to an element in $\operatorname{Ext}^{0}\left(\mathrm{BP}_{*}, \mathrm{BP}_{*} /\left(2^{i+1}, v_{1}^{j}\right)\right)$ (despite the $v_{2}^{-1}$ appearing in its definition) which in turn leads to the element $\beta_{s \cdot 2^{n} / j, i+1}$.

For the second row of the diagram, recall from Hirzebruch's book [7] that, for each level $N>1$, there is a complex genus taking values in the ring of modular forms for the congruence subgroup $\Gamma_{1}(N) \subset \operatorname{SL}(2 ; \mathbb{Z})$; furthermore, as explained by the work of Franke [6], these genera give rise to complex oriented elliptic (co-)homology theories. Thus, working locally at the prime $p$, the orientation yields a map of coefficient rings $\mathrm{BP}_{*} \rightarrow E_{*}^{\Gamma_{1}(N)}$ and induces the upper vertical arrows.

The composition of the vertical arrows on the right-hand side can now be chosen to account for the algebraic portion of Laures' $f$-invariant [9], ie chosen to capture the $p$-local information of the second map in the factorization

$$
f: \pi_{2 k} S^{0} \rightarrow \mathrm{Ext}^{2,2 k+2}\left(\mathrm{MU}_{*}, \mathrm{MU}_{*}\right) \rightarrow \underline{D}_{k+1}^{\Gamma_{1}(N)} \otimes \mathbb{Q} / \mathbb{Z} .
$$

So, in order to compute the $f$-invariant of a member of the beta family, we chase its preimage through the composition of the vertical arrow on the left-hand side with the dotted arrow; put differently, we carry out (a sufficiently large portion of) an elliptic analog of the Greek letter construction explicitly. The result can be summarized as follows (where, as usual, we abbreviate $\beta_{k / j}=\beta_{k / j, 1}$ and $\beta_{k}=\beta_{k / 1}$ ):

Theorem 1 The $f$-invariants of the beta elements of order two are given as follows:

(i) For odd $s \geq 3, \quad f\left(\beta_{s}\right) \equiv \frac{1}{2}\left(\frac{E_{1}^{2}-1}{4}\right)^{s} \bmod \underline{\underline{D}}_{3 s-1}^{\Gamma_{1}(3)}$.

(ii) For odd $s \geq 1, \quad f\left(\beta_{2 s / j}\right) \equiv \frac{1}{2}\left(\frac{E_{1}^{2}-1}{4}\right)^{2 s} \bmod \underline{\underline{D}}_{6 s-j}^{\Gamma_{1}(3)}$.

(iii) For $l \geq 0$ and odd $s \geq 1$,

$$
\begin{aligned}
f\left(\beta_{4 s \cdot 2^{l} / j}\right) & \equiv \frac{1}{2}\left(\frac{E_{1}^{2}-1}{4}\right)^{4 s \cdot 2^{l}}+\frac{1}{2}\left(\frac{E_{1}^{2}-1}{4}\right)^{(4 s-1) 2^{l}} \bmod \underline{D}_{12 s \cdot 2^{l}-j}^{\Gamma_{1}(3)} \\
& \equiv \frac{1}{2}\left(\frac{E_{1}^{2}-1}{4}\right)^{4 s \cdot 2^{l}} \quad \text { if } j \leq 3 \cdot 2^{l}
\end{aligned}
$$


Theorem 2 The $f$-invariants of the beta elements of higher order are given as follows:

(i) For odd $s \geq 1, \quad f\left(\beta_{4 s / 2,2}\right) \equiv \frac{1}{4}\left(\frac{E_{1}^{2}-1}{4}\right)^{4 s} \bmod \underline{\underline{D}}_{12 s-2}^{\Gamma_{1}(3)}$.

(ii) For $l \geq 0, i \geq 1, j=m \cdot 2^{i} \leq a_{l+2}$, odd $s \geq 1$, and modulo $\underline{\underline{D}}_{3 s \cdot 2^{l+i+2}-j}^{\Gamma_{1}(3)}$,

$$
\begin{aligned}
f\left(\beta_{s \cdot 2^{l+i+2} / j, i+1}\right) & \equiv \frac{1}{2^{i+1}}\left(\frac{E_{1}^{2}-1}{4}\right)^{s \cdot 2^{l+i+2}}+\frac{1}{2}\left(\frac{E_{1}^{2}-1}{4}\right)^{\left(s \cdot 2^{i+2}-1\right) 2^{l}} \\
& \equiv \frac{1}{2^{i+1}}\left(\frac{E_{1}^{2}-1}{4}\right)^{s \cdot 2^{l+i+2}} \quad \text { if } j \leq 3 \cdot 2^{l} .
\end{aligned}
$$

(iii) For $k \geq 2, \quad f\left(\beta_{4 k / 2,3}\right) \equiv \frac{1+4 k}{8}\left(\frac{E_{1}^{2}-1}{4}\right)^{4 k} \bmod \underline{\underline{D}}_{12 k-2}^{\Gamma_{1}(3)}$.

(iv) For $l \geq 0, i \geq 1, j=m \cdot 2^{i} \leq a_{l+2}$, odd $s \geq 1$, and modulo $\underline{\underline{D}}_{3 s^{\prime} \cdot 2^{l+i+3}-j}^{\Gamma_{1}(3)}$,

$$
\begin{aligned}
f\left(\beta_{s \cdot 2^{l+i+3} / j, i+2}\right) & \equiv \frac{1}{2^{i+2}}\left(\frac{E_{1}^{2}-1}{4}\right)^{s \cdot 2^{l+i+3}}+\frac{1}{2}\left(\frac{E_{1}^{2}-1}{4}\right)^{\left(s \cdot 2^{i+3}-1\right) 2^{l}} \\
& \equiv \frac{1}{2^{i+2}}\left(\frac{E_{1}^{2}-1}{4}\right)^{s \cdot 2^{l+i+3}} \quad \text { if } j \leq 3 \cdot 2^{l} .
\end{aligned}
$$

The proof presented in the following section turns out to be a pretty straightforward calculation: After a brief recollection of the relevant definitions, we study the image (under the orientation underlying the Hirzebruch genus) of the elements $x_{i}$ and $y_{i}$ occurring in the definition of the beta elements. Then, we sketch our approach to the argument given in [2, Section 4], ie we explain how to carry out the Greek letter construction on the level of (holomorphic) modular forms. The final step consists of performing this computation explicitly.

\section{Proof of the theorems}

\subsection{Preliminaries}

Working with the congruence subgroup $\Gamma_{1}(N) \subset \operatorname{SL}(2 ; \mathbb{Z})$ for a fixed level $N>1$, modular forms will be thought of in terms of their $q$-expansions at the cusp $i \infty$, where $q=e^{2 \pi i \tau}$. Setting $\mathbb{Z}^{\Gamma_{1}(N)}=\mathbb{Z}\left[\zeta_{N}, 1 / N\right]$, where $\zeta_{N}=e^{2 \pi i / N}$, we then denote by $M_{*}^{\Gamma_{1}(N)}$ the graded ring of modular forms with respect to $\Gamma_{1}(N)$ which expand integrally, ie which lie in $\mathbb{Z}^{\Gamma_{1}(N)} \llbracket q \rrbracket$. Now recall from [7, Section 7] that the 
power series associated to the Hirzebruch elliptic genus of level $N$ may be expressed as

$$
Q^{\Gamma_{1}(N)}(x)=x \frac{\Phi(\tau, x-2 \pi i / N)}{\Phi(\tau, x) \Phi(\tau,-2 \pi i / N)},
$$

where the $\Phi$-function is given by

$$
\Phi(\tau, z)=2 \sinh (z / 2) \prod_{n \geq 1} \frac{\left(1-e^{z} q^{n}\right)\left(1-e^{-z} q^{n}\right)}{\left(1-q^{n}\right)^{2}} .
$$

By the splitting principle, the power series (5) determines a homomorphism

$$
\phi^{\Gamma_{1}(N)}: \mathrm{MU}_{*} \rightarrow M_{*}^{\Gamma_{1}(N)},
$$

where $\mathrm{MU}_{*}$ is the coefficient ring of the complex cobordism spectrum, and integrality of the image follows by noting that each term in the $q$-expansion corresponds to a twisted Todd genus. Furthermore, as mentioned in the introduction, the Hirzebruch elliptic genus can be used to construct periodic complex oriented (co-)homology theories [6]: upon inverting, for example, the discriminant form, the Landweber exact functor theorem applies.

Finally, let us explain the map (4) in more detail. To this end, recall from [9] that the ring of divided congruences $D^{\Gamma_{1}(N)}$ consists of those rational combinations of modular forms which expand integrally, and that this ring can be filtered by setting

$$
D_{k+1}^{\Gamma_{1}(N)}=\left\{f=\sum_{i=0}^{k+1} f_{i} \mid f_{i} \in M_{i}^{\Gamma_{1}(N)} \otimes \mathbb{Q}, f \in \mathbb{Z}^{\Gamma_{1}(N)} \llbracket q \rrbracket\right\} ;
$$

furthermore, we put

$$
\underline{D}_{k+1}^{\Gamma_{1}(N)}=D_{k+1}^{\Gamma_{1}(N)}+M_{0}^{\Gamma_{1}(N)} \otimes \mathbb{Q}+M_{k+1}^{\Gamma_{1}(N)} \otimes \mathbb{Q} .
$$

Temporarily switching to the ANSS based on MU, an element in the stable stems of positive even dimension is in second filtration; thus, it can be projected to the 2-line of the $E_{\infty}$-page which in turn injects into the 2-line of the $E_{2}$-page (as there are no differentials hitting it). This explains the first part of the map (4). For the second part, we regard $\mathrm{Ext}^{2, *}\left(\mathrm{MU}_{*}, \mathrm{MU}_{*}\right)$ as a subquotient of $\left(\mathrm{MU}_{*} \otimes \mathbb{Q}\right)^{\otimes 2}$ and consider a representative of an element; under a similar identification, its image under the orientation (determined by the Hirzebruch genus) is represented by a sum of tensor products of modular forms. This sum becomes a rational combination of modular forms (hence an element in the rationalized ring of divided congruences) by replacing, say, each second factor in the sum by the constant term in its $q$-expansion. Working locally at a prime $p \nmid N$, the induced composite map is injective by the results of [9], 
completing the definition of the $f$-invariant; on the other hand, switching back to BP, we arrive at the right-hand side of our diagram (3).

\subsection{The image under the orientation}

Henceforth, we fix $p=2$ and $N=3$, abbreviating $\Gamma=\Gamma_{1}(3)$. Then the ring of modular forms is given by (see eg [8, Section 3.2])

$$
M_{*}^{\Gamma}=\mathbb{Z}^{\Gamma}\left[E_{1}, E_{3}\right]
$$

where

$$
E_{1}=1+6 \sum_{n=1}^{\infty} \sum_{d \mid n}\left(\frac{d}{3}\right) q^{n}, \quad E_{3}=1-9 \sum_{n=1}^{\infty} \sum_{d \mid n}\left(\frac{d}{3}\right) d^{2} q^{n}
$$

are the odd Eisenstein series of the indicated weight at the level $N=3$ (and $(\dot{\div})$ denotes the Legendre symbol); in passing, we note that $\left(E_{1}^{3}-E_{3}\right) / 27 \in \mathbb{Z} \llbracket q \rrbracket$. Furthermore, the following basic congruence can be read off from the $q$-expansions:

$$
E_{3}-1 \equiv \frac{E_{1}^{2}-1}{4} \bmod 2 D_{3}^{\Gamma}
$$

Returning to the Hirzebruch elliptic genus, one may use [7, Appendix I, Theorem 6.2] (see also [3, Appendix C]) to verify that the first few terms of the power series (5), when expressed in terms of the generators $E_{1}$ and $E_{3}$ of $M_{*}^{\Gamma}$, read:

$$
\begin{aligned}
Q^{\Gamma}(x)=1 & +\frac{i E_{1}}{2 \sqrt{3}} x+\frac{E_{1}^{2}}{12} x^{2}+\frac{i E_{1}^{3}-i E_{3}}{18 \sqrt{3}} x^{3}+\frac{13 E_{1}^{4}-16 E_{1} E_{3}}{2160} x^{4} \\
& +\frac{i E_{1}^{2}\left(E_{1}^{3}-E_{3}\right)}{216 \sqrt{3}} x^{5}+\frac{121 E_{1}^{6}-152 E_{1}^{3} E_{3}+40 E_{3}^{2}}{272160} x^{6} \\
& +\frac{i E_{1}}{\sqrt{3}} \frac{7 E_{1}^{6}-11 E_{1}^{3} E_{3}+4 E_{3}^{2}}{19440} x^{7}+O\left(x^{8}\right) .
\end{aligned}
$$

Thus, the genus of the following complex projective spaces is readily evaluated:

$$
\begin{aligned}
& w_{1}=\phi^{\Gamma}\left(\mathbb{C} \mathbb{P}^{1}\right)=\frac{i}{\sqrt{3}} E_{1}, \\
& w_{3}=\phi^{\Gamma}\left(\mathbb{C} \mathbb{P}^{3}\right)=\frac{i}{\sqrt{3}} \frac{5 E_{1}^{3}-2 E_{3}}{9}, \\
& w_{7}=\phi^{\Gamma}\left(\mathbb{C} \mathbb{P}^{7}\right)=\frac{i}{\sqrt{3}} \frac{70 E_{1}^{4} E_{3}-14 E_{1} E_{3}^{2}-65 E_{1}^{7}}{243} .
\end{aligned}
$$

We remind the reader that Hazewinkel's generators of $\mathrm{BP}_{*}$ are recursively defined, and that they are in fact integral, ie they live in $\mathrm{MU}_{*}$ (see eg [11, Appendix A2]). The 
same recursive procedure can be used to determine their respective images under the Hirzebruch genus, which, by abuse of notation, we still denote by $v_{i}$, leading to

$$
\begin{aligned}
& v_{1}=w_{1}=\frac{i}{\sqrt{3}} E_{1}, \\
& v_{2}=\frac{w_{3}-w_{1}^{3}}{2}=\frac{i}{\sqrt{3}} \frac{4 E_{1}^{3}-E_{3}}{9}, \\
& v_{3}=\frac{w_{7}}{4}-\frac{w_{1}^{7}+w_{1} w_{3}^{2}}{8}=\frac{i E_{1}}{\sqrt{3}} \frac{5 E_{1}^{3} E_{3}-E_{3}^{2}-4 E_{1}^{6}}{81} ;
\end{aligned}
$$

in particular, we see that $v_{3}$ becomes decomposable in $M_{*}^{\Gamma}$ :

$$
\begin{aligned}
v_{3} & =\frac{i E_{1}}{\sqrt{3}}\left(\frac{4 E_{1}^{3} E_{3}-E_{3}^{2}}{81}-\frac{4 E_{1}^{6}-E_{1}^{3} E_{3}}{81}\right) \\
& =\frac{i E_{1}}{\sqrt{3}}\left(\frac{i}{\sqrt{3}} \frac{4 E_{1}^{3}-E_{3}}{9}\right)\left(-\frac{i}{3 \sqrt{3}}\left(E_{3}-E_{1}^{3}\right)\right) \\
& =3 v_{1} v_{2}\left(v_{2}+v_{1}^{3}\right) .
\end{aligned}
$$

Continuing with our abuse of notation, we now consider the $x_{i}$ as elements in $v_{2}^{-1} M_{*}^{\Gamma}$, where, due to (7), their original definition $[12,(1.1)]$ simplifies to

$$
\begin{aligned}
& x_{0}=v_{2}, \\
& x_{1}=v_{2}^{2}-v_{1}^{2} v_{2}^{-1} v_{3}=v_{2}^{2}-3 v_{1}^{3}\left(v_{2}+v_{1}^{3}\right), \\
& x_{2}=x_{1}^{2}-v_{1}^{3} v_{2}^{3}-v_{1}^{5} v_{3}=v_{2}^{4}-7 v_{1}^{3} v_{2}^{3}+15 v_{1}^{9} v_{2}+9 v_{1}^{12}, \\
& x_{i}=x_{i-1}^{2} \quad i \geq 3,
\end{aligned}
$$

showing that the (images of the) $x_{i}$ are actually holomorphic. On the other hand, unless $i=0$, this is not true for the $y_{i} \in v_{1}^{-1} M_{*}^{\Gamma}$, which read:

$$
\begin{aligned}
& y_{0}=v_{1}, \\
& y_{1}=v_{1}^{2}-4 v_{1}^{-1} v_{2}, \\
& y_{i}=y_{i-1}^{2}, \quad i \geq 2 .
\end{aligned}
$$

However, for $i \geq 1$ and $m \geq 1$, we may introduce

$$
z_{i, m}=v_{1}^{m \cdot 2^{i}}-m \cdot 2^{i+1} v_{1}^{m \cdot 2^{i}-3} v_{2}
$$


which are holomorphic for $m \cdot 2^{i} \geq 4$ and satisfy

$$
\begin{aligned}
z_{i, m} & \equiv y_{i}^{m} & & \bmod 2^{i+2} v_{1}^{-1} M_{*}^{\Gamma} \\
& \equiv 1 & & \bmod 2^{i+2} \mathbb{Z}^{\Gamma} \llbracket q \rrbracket,
\end{aligned}
$$

the second line being an immediate consequence of (6).

\subsection{Determining "elliptic" beta elements}

Requiring $p>3$ and working with the full modular group, Behrens and Laures have shown in [2, Section 4] how an element in $\operatorname{Ext}^{0}\left(M_{*}, M_{*} /\left(p^{\infty}, E_{p-1}^{\infty}\right)\right)$ gives rise to an element in $D \otimes \mathbb{Q} / D\left[\frac{1}{6}\right]+M_{k} \otimes \mathbb{Q}+\mathbb{Q}$; clearly, the other primes can be treated analogously by working with a smaller congruence subgroup. Let us rephrase their argument in a language closer to the original formulation of the Greek letter construction:

Still working at the prime $p=2$ and the level $N=3$, we choose a (holomorphic) modular form $\mu \in M_{|\mu|}^{\Gamma}$ and a pair of positive integers $\left(i_{0}, i_{1}\right)$ such that

$$
\mu^{i_{1}} \equiv 1 \quad \bmod 2^{i_{0}} D_{i_{1}|\mu|}^{\Gamma}
$$

in particular, this ensures that $\left(2^{i_{0}}, \mu^{i_{1}}\right)$ is regular on $M_{*}^{\Gamma}$.

Now, given a modular form $\tilde{\varphi}_{t} \in M_{t}^{\Gamma}$, we can use the natural inclusion

$$
M_{t}^{\Gamma} \hookrightarrow D_{t}^{\Gamma}
$$

and ask whether $\tilde{\varphi}_{t}$ satisfies

$$
\tilde{\varphi}_{t} \equiv \mu^{i_{1}} \varphi_{t / i_{1}|\mu|, i_{0}} \quad \bmod 2^{i_{0}} D_{t}^{\Gamma}
$$

for some

$$
\varphi_{t / i_{1}|\mu|, i_{0}} \in D_{t-i_{1}|\mu|}^{\Gamma} / 2^{i_{0}} D_{t-i_{1}|\mu|}^{\Gamma}
$$

Let us call a modular form satisfying (10) invariant mod $\left(2^{i_{0}}, \mu^{i_{1}}\right)$. Moreover, we have the obvious composition:

$$
\stackrel{(\cdot)}{=} D_{k}^{\Gamma} / 2^{i_{0}} D_{k}^{\Gamma} \cong D_{k}^{\Gamma} \otimes \mathbb{Z} / 2^{i_{0}} \rightarrow D_{k}^{\Gamma} \otimes \mathbb{Q} / \mathbb{Z} \rightarrow \underline{\underline{D}}_{k}^{\Gamma} \otimes \mathbb{Q} / \mathbb{Z}, \quad \varphi_{k} \mapsto \underline{\underline{\varphi}}_{k} .
$$

Then it is easy to see that, for a modular form $\tilde{\varphi}_{t}$ satisfying (10), the assignment

$$
\tilde{\varphi}_{t} \mapsto \underline{\underline{\varphi}} t / i_{1}|\mu|, i_{0}
$$


depends only on the reduction of $\tilde{\varphi}_{t} \bmod \left(2^{i_{0}}, \mu^{i_{1}}\right)$, hence descends to a well-defined map

$$
\operatorname{ker}\left(M_{t}^{\Gamma} /\left(2^{i_{0}}, \mu^{i_{1}}\right) \rightarrow D_{t}^{\Gamma} /\left(2^{i_{0}}, \mu^{i_{1}}\right)\right) \rightarrow \underline{\underline{D}}_{t-i_{1}|\mu|}^{\Gamma} \otimes \mathbb{Q} / \mathbb{Z}
$$

which we may think of as the "elliptic" Greek letter beta map and which corresponds to the dotted arrow in our diagram (3). More precisely, by removing the constant term of the $q$-expansion, we obtain another map

$$
d: M_{t}^{\Gamma} \rightarrow D_{t}^{\Gamma}, \quad d\left(\widetilde{\varphi}_{t}\right)=\widetilde{\varphi}_{t}-q^{0}\left(\widetilde{\varphi}_{t}\right),
$$

which might look like a more natural choice with respect to which invariance should be defined, see [2, Section 4]. However, we have $q^{0}\left(\widetilde{\varphi}_{t}\right) \equiv \mu^{i_{1}} q^{0}\left(\widetilde{\varphi}_{t}\right) \bmod 2^{i_{0}} D_{t}^{\Gamma}$, hence both choices agree up to a shift of $\varphi_{t / i_{1}|\mu|, i_{0}}$ by the constant $q^{0}\left(\widetilde{\varphi}_{t}\right)$; as the latter maps to zero in $\underline{\underline{D}}_{t-i_{1}|\mu|}^{\Gamma} \otimes \mathbb{Q} / \mathbb{Z}$, our construction is visibly equivalent to the one leading to [2, Theorem 4.2].

\subsection{Explicit computations}

Computing the effect of the elliptic Greek letter map (11) on the preimage of Shimomura's beta elements now amounts to exhibiting appropriate congruences; the elements defined by (1) are dealt with easily, since $\left(2^{i+1}, v_{1}^{j}\right)$ is regular on $M_{*}^{\Gamma}$ provided that $j=m \cdot 2^{i}$; moreover, for $k \geq 0$ this implies:

$$
\left(\frac{E_{1}^{2}-1}{4}\right)^{k} \equiv v_{1}^{j}\left(\frac{E_{1}^{2}-1}{4}\right)^{k} \bmod 2^{i+1} D_{2 k+j}^{\Gamma}
$$

Furthermore, the following two results are useful:

Lemma 3 For $i \geq 0, l \geq 0, m \cdot 2^{i}=j \leq 6 \cdot 2^{l}$, we have

$$
E_{3}^{s \cdot 2^{l+i+2}} \equiv\left(\frac{E_{1}^{2}-1}{4}\right)^{s \cdot 2^{l+i+2}} \bmod 2^{i+1} D_{12 s \cdot 2^{l+i}}^{\Gamma}+v_{1}^{j} \cdot M_{12 s \cdot 2^{l+i}-j}^{\Gamma} .
$$

Proof It is easy to see that for $l \geq 0$ and $i \geq 0$, we have

$$
E_{3}^{2^{l+i+2}} \equiv\left(E_{3}-v_{1}^{3}\right)^{2^{l+i+2}}+2^{i+1}\left(v_{1}^{6} E_{3}^{2}\right)^{2^{l}} E_{3}^{2^{l+2}\left(2^{i}-1\right)} \bmod \left(2^{i+2}, v_{1}^{12 \cdot 2^{l}}\right),
$$

and the basic congruence (6) implies

$$
\left(E_{3}-v_{1}^{3}\right)^{2^{k}} \equiv\left(\frac{E_{1}^{2}-1}{4}\right)^{2^{k}} \bmod 2^{k+1} D_{3 \cdot 2^{k}}^{\Gamma}
$$

This concludes the proof. 
Lemma 4 For $i \geq 0, l \geq 0,1 \leq j \leq 6 \cdot 2^{l}$, we have

$$
\begin{aligned}
E_{3}^{\left(s \cdot 2^{i+2}-1\right) 2^{l}} & \equiv\left(\frac{E_{1}^{2}-1}{4}\right)^{\left(s \cdot 2^{i+2}-1\right) 2^{l}} & & \bmod 2 D_{12 s \cdot 2^{l+i}}^{\Gamma}+v_{1}^{j} \cdot M_{12 s \cdot 2^{l+i}-j}^{\Gamma} \\
& \equiv 0 & & \text { if } j \leq 3 \cdot 2^{l} .
\end{aligned}
$$

Proof Noting that

$$
\begin{aligned}
& E_{3}^{\left(s \cdot 2^{i+2}-1\right) 2^{l}} \equiv v_{1}^{3 \cdot 2^{l}}\left(E_{3}-v_{1}^{3}\right)^{\left(s \cdot 2^{i+2}-1\right) 2^{l}} \quad \bmod 2 D_{12 s \cdot 2^{l+i}}^{\Gamma}+v_{1}^{j} \cdot M_{12 s \cdot 2^{l+i}-j}^{\Gamma} \\
& \equiv\left(E_{3}-1\right)^{\left(s \cdot 2^{i+2}-1\right) 2^{l}} \quad \bmod 2 D_{12 s \cdot 2^{l+i}}^{\Gamma}+v_{1}^{j} \cdot M_{12 s \cdot 2^{l+i}-j}^{\Gamma},
\end{aligned}
$$

the claim follows from (6).

Proof of Theorem 1 For part (i), we observe that

$$
\begin{array}{rlr}
x_{0}^{s} & =v_{2}^{s} & \\
& \equiv E_{3}^{s} & \bmod 2 D_{3 s}^{\Gamma} \\
& \equiv\left(E_{3}-v_{1}^{3}\right)^{s} & \bmod 2 D_{3 s}^{\Gamma}+v_{1} \cdot M_{3 s-1}^{\Gamma} \\
& \equiv\left(\frac{E_{1}^{2}-1}{4}\right)^{s} & \bmod 2 D_{3 s}^{\Gamma}+v_{1} \cdot M_{3 s-1}^{\Gamma} .
\end{array}
$$

Similarly, for part (ii) we have:

$$
\begin{aligned}
x_{1}^{s} & \equiv v_{2}^{2 s} & & \bmod v_{1}^{j} \\
& \equiv E_{3}^{2 s} & & \bmod 2 D_{6 s}^{\Gamma}+v_{1}^{j} \cdot M_{6 s-j}^{\Gamma} \\
& \equiv\left(E_{3}-v_{1}^{3}\right)^{2 s} & & \bmod 2 D_{6 s}^{\Gamma}+v_{1}^{j} \cdot M_{6 s-j}^{\Gamma} \\
& \equiv\left(\frac{E_{1}^{2}-1}{4}\right)^{2 s} & & \bmod 2 D_{6 s}^{\Gamma}+v_{1}^{j} \cdot M_{6 s-j}^{\Gamma},
\end{aligned}
$$

and since $j \leq a_{l+2}=6 \cdot 2^{l}$ (and $j \leq 2^{l+2}$ if $s=1$ ), for part (iii) we conclude

$$
\begin{aligned}
x_{2+l}^{s} & \equiv v_{2}^{4 s \cdot 2^{l}}+v_{1}^{3 \cdot 2^{l}} v_{2}^{(4 s-1) 2^{l}} & & \bmod \left(2, v_{1}^{a_{l+2}}\right) \\
& \equiv E_{3}^{4 s \cdot 2^{l}}+E_{3}^{(4 s-1) 2^{l}} & & \bmod 2 D_{12 s \cdot 2^{l}}^{\Gamma}+v_{1}^{j} \cdot M_{12 s \cdot 2^{l}-j}^{\Gamma} \\
& \equiv\left(\frac{E_{1}^{2}-1}{4}\right)^{4 s \cdot 2^{l}}+\left(\frac{E_{1}^{2}-1}{4}\right)^{(4 s-1) 2^{l}} & & \bmod 2 D_{12 s \cdot 2^{l}}^{\Gamma}+v_{1}^{j} \cdot M_{12 s \cdot 2^{l}-j}^{\Gamma} .
\end{aligned}
$$

In view of (12), this completes the proof. 
Remark 5 Since $x_{0}=v_{2}$ is sent to zero under the map (11) with respect to $\left(2, v_{1}\right)$, we see that in order to obtain something interesting, we have to impose $s \geq 3$ in part (i). In a similar vein, the condition $j \leq 2^{l+2}$ if $s=1$ in part (iii) is needed to ensure that $D_{8 s \cdot 2^{l}+j}^{\Gamma} \subset D_{12 s \cdot 2^{l}}^{\Gamma}$ when using (12).

Now we turn our attention to the elements $\beta_{4 s \cdot 2^{l} / j, i+1}$ for $i \geq 1$.

Proof of Theorem 2(i) The choice $n=2$ and $i=1$ in (1) dictates $j=2$, hence we compute

$$
\begin{aligned}
x_{2}^{s} & \equiv v_{2}^{4 s} & & \bmod \left(4, v_{1}^{2}\right) \\
& \equiv E_{3}^{4 s} & & \bmod 4 D_{12 s}^{\Gamma}+v_{1}^{2} \cdot M_{12 s-2}^{\Gamma} \\
& \equiv\left(\frac{E_{1}^{2}-1}{4}\right)^{4 s} & & \bmod 4 D_{12 s}^{\Gamma}+v_{1}^{2} \cdot M_{12 s-2}^{\Gamma}
\end{aligned}
$$

Combined with (12), this yields the claim.

Lemma 6 For $l \geq 0$ and $i \geq 0$, we have

$$
x_{l+i+3} \equiv v_{2}^{2^{l+i+3}}+2^{i+1} v_{1}^{3 \cdot 2^{l}} v_{2}^{\left(2^{i+3}-1\right) 2^{l}} \bmod \left(2^{i+2}, v_{1}^{a_{l+2}}\right) .
$$

Proof Since $(a+b)^{2^{l+1}} \equiv a^{2^{l+1}}+b^{2^{l+1}}+2(a b)^{2^{l}} \bmod 4$ for $l \geq 0$, we compute

$$
x_{l+3}=x_{2}^{2^{l+1}} \equiv v_{2}^{8 \cdot 2^{l}}+2\left(v_{1}^{3} v_{2}\right)^{2^{l}} v_{2}^{6 \cdot 2^{l}} \bmod \left(4, v_{1}^{a_{l+2}}\right)
$$

and use the binomial theorem.

Proof of Theorem 2(ii) In order to treat the remaining cases of our computation of $x_{n}^{s} \bmod \left(2^{i+1}, v_{1}^{j}\right)$, we notice that since (1) requires $j=m \cdot 2^{i} \leq a_{n-i}$, and since all cases with $i=0$ and the case $i=1$ for $n=2$ have already been taken care of, it suffices to consider $n=l+i+2$ where $l \geq 0$ and $i \geq 1$; now, for odd $s \geq 1$ we have (by Lemma 6 in a reindexed form)

$$
\begin{array}{rlrl}
x_{l+i+2}^{s} & \equiv v_{2}^{s \cdot 2^{l+i+2}}+2^{i} v_{1}^{3 \cdot 2^{l}} v_{2}^{s \cdot 2^{l+i+2}-2^{l}} & \bmod \left(2^{i+1}, v_{1}^{a_{l+2}}\right) \\
& \equiv E_{3}^{s \cdot 2^{l+i+2}}+2^{i} E_{3}^{s \cdot 2^{l+i+2}-2^{l}} & & \bmod 2^{i+1} D_{12 s \cdot 2^{l+i}}^{\Gamma}+v_{1}^{j} \cdot M_{12 s \cdot 2^{l+i}-j}^{\Gamma},
\end{array}
$$

from which the desired result follows.

Finally, we treat the beta elements defined by (2): 
Proof of Theorem 2(iii) In order to compute the $f$-invariant of $\beta_{4 k / 2,3}$, we are going to show that, although $z_{1,1}=y_{1}=v_{1}^{2}-4 v_{1}^{-1} v_{2}$ is not holomorphic, we can still make sense out of the map (11) with respect to $\left(8, z_{1,1}\right)$ if $t=12 k \geq 24$. To this end, we observe

$$
v_{1}^{6}=z_{1,1} v_{1}^{4}+4 v_{1}^{3} v_{2}=z_{1,1}\left(v_{1}^{4}+4 v_{1} v_{2}\right)+16 v_{2}^{2},
$$

hence we compute

$$
\begin{aligned}
x_{2}^{k} & \equiv v_{2}^{4 k}+k v_{1}^{3} v_{2}^{4 k-1} & & \bmod \left(8, v_{1}^{6}\right) \\
& \equiv(1+4 k) v_{2}^{4 k} & & \bmod \left(8, z_{1,1}\right) \\
& \equiv(1+4 k) E_{3}^{4 k} & & \bmod 8 D_{12 k}^{\Gamma}+z_{1,1} \cdot M_{12 k-2}^{\Gamma},
\end{aligned}
$$

where $z_{1,1} \cdot M_{12 k-2}^{\Gamma} \subset M_{12 k}^{\Gamma}$ for dimensional reasons. Finally, we note that

$$
\begin{array}{rlrl}
E_{3}^{4 k} & \equiv\left(\frac{E_{1}^{2}-1}{4}\right)^{4 k} \quad \bmod 8 D_{12 k}^{\Gamma}+z_{1,1} \cdot M_{12 k-2}^{\Gamma} & \\
& \equiv\left(\frac{E_{1}^{2}-1}{4}\right)^{4 k} v_{1}^{4} z_{1,1} \quad \bmod 8 D_{12 k}^{\Gamma}+z_{1,1} \cdot M_{12 k-2}^{\Gamma} \quad \text { if } k \geq 2 ;
\end{array}
$$

as $v_{1}^{4} \equiv 1 \bmod 8$, the claim follows.

Proof of Theorem 2(iv) Recall that in the definition (2) we have to impose $j=$ $m \cdot 2^{i} \leq a_{n-i-l}$ for $n \geq 3$; since the situation $m=i=1$ has already been dealt with in the previous part (iii), it is sufficient to consider the case $n=l+i+3$, $4 \leq m \cdot 2^{i}=j \leq a_{l+2}$, where $l \geq 0, i \geq 1$. In order to compute the $f$-invariants, we calculate the effect of the map (11) with respect to $\left(2^{i+2}, z_{i, m}\right)$. Since

$$
\begin{aligned}
& v_{1}^{6 \cdot 2^{l}}=z_{i, m} v_{1}^{6 \cdot 2^{l}-j}+2 j v_{1}^{6 \cdot 2^{l}-3} v_{2}, \\
& v_{1}^{9 \cdot 2^{l}}=z_{i, m}\left(v_{1}^{9 \cdot 2^{l}-j}+2 j v_{1}^{9 \cdot 2^{l}-j-3} v_{2}\right)+4 j^{2} v_{1}^{9 \cdot 2^{l}-6} v_{2}^{2},
\end{aligned}
$$

we calculate, for $l \geq 0, i \geq 1$, and odd $s \geq 1$,

$$
\begin{array}{ll}
x_{l+i+3}^{s} & \\
\equiv v_{2}^{s \cdot 2^{l+i+3}}+2^{i+1} v_{1}^{3 \cdot 2^{l}} v_{2}^{\left(s 2^{i+3}-1\right) 2^{l}}+3 s \cdot 2^{i} v_{1}^{6 \cdot 2^{l}} v_{2}^{\left(s 2^{i+3}-2\right) 2^{l}} & \bmod \left(2^{i+2}, v_{1}^{9 \cdot 2^{l}}\right) \\
\equiv v_{2}^{s \cdot 2^{l+i+3}}+2^{i+1} v_{1}^{3 \cdot 2^{l}} v_{2}^{\left(s 2^{i+3}-1\right) 2^{l}} & \bmod \left(2^{i+2}, z_{i, m}\right),
\end{array}
$$

hence

$x_{l+i+3}^{s} \equiv E_{3}^{s \cdot 2^{l+i+3}}+2^{i+1} E_{3}^{\left(s \cdot 2^{i+3}-1\right) 2^{l}} \bmod 2^{i+2} D_{24 s \cdot 2^{l+i}}^{\Gamma}+z_{i, m} \cdot M_{24 s \cdot 2^{l+i}-j}^{\Gamma}$. 
Furthermore, the proof of Lemma 3 shows

$$
\begin{aligned}
& E_{3}^{2^{l+(i+1)+2}} \equiv \\
& \quad\left(E_{3}-v_{1}^{3}\right)^{2^{l+(i+1)+2}}+2^{(i+1)+1}\left(v_{1}^{6} E_{3}^{2}\right)^{2^{l}} E_{3}^{2^{l+2}}\left(2^{i+1}-1\right) \bmod \left(2^{(i+1)+2}, v_{1}^{12 \cdot 2^{l}}\right) .
\end{aligned}
$$

Thus, it follows that

$$
E_{3}^{2^{l+(i+1)+2}} \equiv\left(E_{3}-v_{1}^{3}\right)^{2^{l+(i+1)+2}} \bmod \left(2^{i+2}, v_{1}^{12 \cdot 2^{l}}\right),
$$

and due to (13), application of Lemma 3 and Lemma 4 yields the claim.

Acknowledgements The author would like to thank Matthew Ando and an anonymous referee for comments that helped to improve the exposition of this article.

\section{References}

[1] M Behrens, Congruences between modular forms given by the divided $\beta$ family in homotopy theory, Geom. Topol. 13 (2009) 319-357 MR2469520

[2] M Behrens, G Laures, $\beta$-family congruences and the $f$-invariant, from "New topological contexts for Galois theory and algebraic geometry" (A Baker, B Richter, editors), Geom. Topol. Monogr. 16, Coventry (2009) 9-29 MR2544384

[3] H von Bodecker, On the geometry of the $f$-invariant, $\mathrm{PhD}$ thesis (2008) Available at http://tinyurl.com/BodeckerThesis

[4] H von Bodecker, On the $f$-invariant of products, Homology Homotopy Appl. 18 (2016) 169-176

[5] U Bunke, N Naumann, The $f$-invariant and index theory, Manuscripta Math. 132 (2010) 365-397 MR2652438

[6] J Franke, On the construction of elliptic cohomology, Math. Nachr. 158 (1992) 43-65

[7] F Hirzebruch, T Berger, R Jung, Manifolds and modular forms, Aspects of Mathematics E20, Friedr. Vieweg \& Sohn, Braunschweig (1992) MR1189136 With appendices by Nils-Peter Skoruppa and by Paul Baum

[8] J Hornbostel, N Naumann, Beta-elements and divided congruences, Amer. J. Math. 129 (2007) 1377-1402 MR2354323

[9] G Laures, The topological q-expansion principle, Topology 38 (1999) 387-425 MR1660325

[10] H R Miller, D C Ravenel, W S Wilson, Periodic phenomena in the Adams-Novikov spectral sequence, Ann. of Math. 106 (1977) 469-516 MR0458423

[11] D C Ravenel, Complex cobordism and stable homotopy groups of spheres, 2nd edition, AMS Chelsea Series 347, Amer. Math. Soc., Providence, RI (2003)

[12] K Shimomura, Novikov's Ext ${ }^{2}$ at the prime 2, Hiroshima Math. J. 11 (1981) 499-513 MR635034 
Fakultät für Mathematik, Universität Bielefeld

Postfach 100131, D-33501 Bielefeld, Germany

hvb@math.uni-bielefeld.de

Received: 1 June 2015 Revised: 4 January 2016 